PROCEEDINGS OF THE

AMERICAN MATHEMATICAL SOCIETY

Volume 125, Number 11, November 1997, Pages 3363-3370

S 0002-9939(97)04002-1

\title{
PERTURBATIONS OF THE HAAR WAVELET
}

\author{
N. K. GOVIL AND R. A. ZALIK \\ (Communicated by Palle E. T. Jorgensen)
}

\begin{abstract}
Let $m \in Z^{+}$be given. For any $\varepsilon>0$ we construct a function $f^{\{\varepsilon\}}$ having the following properties: (a) $f^{\{\varepsilon\}}$ has support in $[-\varepsilon, 1+\varepsilon]$. (b) $f^{\{\varepsilon\}} \in C^{m}(-\infty, \infty)$. (c) If $h$ denotes the Haar function and $0<\delta<\infty$, then $\left\|f^{\{\varepsilon\}}-h\right\|_{L^{\delta}(\mathcal{R})} \leq\left(1+2^{\delta}\right)^{1 / \delta}(2 \varepsilon)^{1 / \delta}$. (d) $f^{\{\varepsilon\}}$ generates an affine Riesz basis whose frame bounds (which are given explicitly) converge to 1 as $\varepsilon \rightarrow 0$.
\end{abstract}

Let $\mathcal{H}$ be a Hilbert space with inner product $\langle\cdot, \cdot\rangle$ and norm $\|\cdot\|:=\langle\cdot, \cdot\rangle^{1 / 2}$. Let $\mathcal{Z}^{+}$denote the natural numbers. A sequence $\left\{f_{n}, n \in Z^{+}\right\} \subset \mathcal{H}$ is called a frame if there are constants $A$ and $B$ such that for every $f \in \mathcal{H}$

$$
A\|f\|^{2} \leq \sum_{n \in Z^{+}}\left|\left\langle f, f_{n}\right\rangle\right|^{2} \leq B\|f\|^{2} .
$$

The constants $A$ and $B$ are called bounds of the frame. If only the right-hand inequality is satisfied for all $f \in \mathcal{H}$, then $\left\{f_{n}, n \in Z^{+}\right\}$is called a Bessel sequence with bound $B$. A frame is called exact, or a Riesz basis, if upon the removal of any single element of the sequence, it ceases to be a frame. However, not every frame is a Riesz basis: as is well known, a sequence $\left\{f_{n}, n \in Z^{+}\right\} \subset \mathcal{H}$ is a Riesz basis if and only if it is the image of an orthonormal basis under a bounded invertible linear operator $U: \mathcal{H} \rightarrow \mathcal{H}([1,11])$. Clearly for an orthonormal basis both frame bounds equal 1 .

In [5] it is shown that adding a Bessel sequence with a small bound to a Riesz basis transforms the original basis into another Riesz basis. It is also shown how frame bounds for the new basis are obtained from frame bounds of the original basis. Given an arbitrary positive integer $m$, in the present paper we use these results to perturb the Haar function into a function $f^{\{\varepsilon\}} \in C^{m}(-\infty, \infty)$ with support in $[-\varepsilon, 1+\varepsilon]$ (thus having good time and frequency localization) that preserves the symmetry of the Haar wavelet and generates an affine Riesz basis in $L^{2}(-\infty, \infty)$. The lack of orthogonality precludes the use of the fast wavelet transform. On the other hand the functions $f^{\{\varepsilon\}}$ are given explicitly in terms of cardinal $B$-splines. Other wavelets do not have a closed form representation and have to be obtained recursively, using the cascade algorithm $[4,10]$. Since frame bounds are given

Received by the editors March 18, 1996 and, in revised form, June 21, 1996.

1991 Mathematics Subject Classification. Primary 42C99; Secondary 41A05, 46C99.

Key words and phrases. Frames, affine frames, Riesz bases, Haar wavelet, basis perturbations, $\wedge$-bounded mean variation, cardinal splines.

The authors are grateful to Ole Christensen, Sergio J. Favier, Christopher E. Heil, and Luis Miguel Pozo Coronado for their helpful comments. 
explicitly, the frame algorithm or other algorithms can be used to reconstruct a function from its wavelet coefficients $([4,6])$.

Let $\mathcal{Z}$ denote the integers, $\mathcal{R}$ the real numbers, and

$$
\phi_{j, k}(x)=a^{j / 2} \phi\left(a^{j} x-b k\right),
$$

where $a>1$ and $b>0$. Our construction is based on a sequence of auxiliary propositions, some of which are of independent interest. We begin with:

Lemma 1. Let $\phi \in L^{2}(\mathcal{R})$. If

$$
\sum_{j, k \in \mathcal{Z}}\left|\left\langle\phi_{j, k}, \phi_{j^{\prime}, k^{\prime}}\right\rangle\right| \leq B
$$

for every $j^{\prime}, k^{\prime} \in \mathcal{Z}$, then $\left\{\phi_{j, k}\right\}_{j, k \in \mathcal{Z}}$ is a Bessel sequence with bound $B$.

Proof. From Schur's lemma ([3, Lemma 5] or [11, p. 159]) we deduce that the operator generated by $\left\{\left\langle\phi_{j, k}, \phi_{j^{\prime}, k^{\prime}}\right\rangle, j, j^{\prime}, k, k^{\prime} \in \mathcal{Z}\right\}$ is bounded in $\ell^{2}\left(\mathcal{Z}^{2}\right)$, and the assertion follows from the discussion in [11, p. 159].

Remark. This lemma was implicitly used in the proof of [3, Lemma 9]. See also [7, Proposition 9.10].

The next result is a quantitative version of [2, Lemma 9], and its proof is based on the concept of $\wedge$-bounded mean variation introduced by Shi [8]:

Let $\wedge:=\left\{\lambda_{k}, k=1,2, \ldots\right\}$ be a nondecreasing sequence of positive numbers such that $\sum \frac{1}{\lambda_{n}}=\infty$. For any locally integrable function $f$ and a bounded interval $I_{j}$ of length $\left|I_{j}\right|$, define

$$
\begin{gathered}
f_{I_{j}}:=\frac{1}{\left|I_{j}\right|} \int_{I_{j}} f(t) d t, \\
\mu_{I_{j}}(f):=\frac{1}{\left|I_{j}\right|} \int_{I_{j}}\left|f(t)-f_{I_{j}}\right| d t .
\end{gathered}
$$

The function $f$ is said to be of $\wedge$-bounded mean variation on an interval $I$, and we write $f \in \wedge B M V(I)$, if

$$
M_{\wedge}(f, I)=\sup \sum_{j=1}^{\infty} \frac{\mu_{I_{j}}(f)}{\lambda_{j}}<\infty,
$$

where the supremum is taken over all sequences of nonoverlapping subintervals $I_{j}$ of $I$.

Let $B V(\mathcal{R})$ denote the set of functions of bounded variation over $\mathcal{R}$. We have:

Lemma 2. Assume that $\phi$ satisfies the following conditions:

(a) $\operatorname{supp} \phi \subseteq[c, d]=: I$,

(b) $\phi \in B V(\mathcal{R})$,

(c) $\int_{\mathcal{R}} \phi(t) d t=0$.

If $V_{\phi}$ denotes the total variation of $\phi$ on $\mathcal{R}$, then $\left\{\phi_{j, k}\right\}_{j, k \in \mathcal{Z}}$ is a Bessel sequence with Bessel bound

$$
M_{\phi}:=\|\phi\|_{\infty}\left(\frac{\sqrt{a}}{\sqrt{a}-1}\right)(d-c)\left\{\left[(d-c) b^{-1}+1\right] V_{\phi}+\frac{(a+1)}{\sqrt{a}} b^{-1}\|\phi\|_{\infty}\right\} .
$$


Proof. Let $\theta_{j^{\prime}, k^{\prime}}=\sum_{j, k \in \mathcal{Z}}\left|\left\langle\phi_{j, k}, \phi_{j^{\prime}, k^{\prime}}\right\rangle\right|$. Then

$$
\theta_{j^{\prime}, k^{\prime}}=\sum_{j \geq j^{\prime}} \sum_{k \in \mathcal{Z}}+\sum_{j<j^{\prime}} \sum_{k \in \mathcal{Z}}=\Sigma_{1}+\Sigma_{2} .
$$

If $I_{j, k}:=\left\{x: a^{j} x-k b \in(c, d)\right\}=\left(a^{-j}(c+k b), a^{-j}(d+k b)\right), K\left(j, j^{\prime}, k^{\prime}\right):=$ $\left\{k: I_{j, k} \cap I_{j^{\prime}, k^{\prime}} \neq \emptyset\right\}$,

$$
h_{j-j^{\prime}, k}:=\frac{1}{\left|I_{j-j^{\prime}, k}\right|} \int_{I_{j-j^{\prime}, k}} \phi(x-k b) d x,
$$

and

$$
\mu_{I_{j-j^{\prime}, k}}\left(\phi_{0, k^{\prime}}\right):=\frac{1}{\left|I_{j-j^{\prime}, k}\right|} \int_{I_{j-j^{\prime}, k}}\left|\phi_{0, k^{\prime}}(x)-h_{j-j^{\prime}, k}\right| d x,
$$

from $[3,(3.13)]$ we conclude that

$$
\Sigma_{1} \leq\|\phi\|_{\infty}(d-c) \sum_{j-j^{\prime} \geq 0} a^{-\left(j-j^{\prime}\right) / 2} \sum_{k \in K\left(j-j^{\prime}, 0, k^{\prime}\right)} \mu_{I_{j-j^{\prime}, k}}\left(\phi_{0, k^{\prime}}\right) .
$$

As remarked in [3] (following (3.14)),

$$
\sum_{k \in K\left(j-j^{\prime}, 0, k^{\prime}\right)} \chi_{I_{j-j^{\prime}, k}}(x) \leq(d-c) b^{-1}+1 .
$$

Let $I_{n}$ be an arbitrary sequence of non-overlapping intervals in $[c, d]$ and for $n=1,2, \ldots$ let $\alpha_{n}=\inf _{x \in I_{n}} \phi(x)$ and $\beta_{n}=\sup _{x \in I_{n}} \phi(x)$. Then for every $\varepsilon>0$, we can select two points $\gamma_{n}$ and $\delta_{n}$ in each interval $I_{n}$ so that

$$
\phi\left(\gamma_{n}\right) \leq \alpha_{n}+\varepsilon 2^{-n-1}, \quad \phi\left(\delta_{n}\right) \geq \beta_{n}-\varepsilon 2^{-n-1} .
$$

Clearly

Hence by (3),

$$
\mu_{I_{n}}(\phi)=\frac{1}{\left|I_{n}\right|} \int_{I_{n}}\left|\phi(x)-\phi_{I_{n}}\right| d x \leq \beta_{n}-\alpha_{n} .
$$

$$
\begin{aligned}
\sum \mu_{I_{n}}(\phi) & \leq \sum_{n=1}^{\infty}\left|\phi\left(I_{n}^{\prime}\right)\right|+\varepsilon \sum_{n=1}^{\infty} \frac{1}{2^{n}} \\
& \leq V_{\phi}+\varepsilon,
\end{aligned}
$$

where $I_{n}^{\prime}=\left[\gamma_{n}, \delta_{n}\right]$ and $\phi\left(I_{n}^{\prime}\right)=\phi\left(\gamma_{n}\right)-\phi\left(\delta_{n}\right)$.

Since (4) holds for every $\varepsilon>0$, we obtain

$$
\sum \mu_{I_{n}}(\phi) \leq V_{\phi}
$$

implying that $\phi \in \wedge B M V(c, d)$, and

$$
M_{\wedge}(\phi, I) \leq V_{\phi},
$$

with $\wedge:=\left\{\lambda_{k}: k=1,2, \ldots\right\}$ and $\lambda_{k}=1$ for $k=1,2, \ldots$

Thus (2), (6) and [3, Lemma 7] yield

$$
\sum_{k \in K\left(j-j^{\prime}, 0, k^{\prime}\right)} \mu_{I_{j-j^{\prime}, k}}\left(\phi_{0, k^{\prime}}\right) \leq\left[(d-c) b^{-1}+1\right] V_{\phi},
$$

and from (1) we conclude that

$$
\Sigma_{1} \leq\|\phi\|_{\infty}(d-c) \frac{\sqrt{a}}{\sqrt{a}-1}\left[(d-c) b^{-1}+1\right] V_{\phi} .
$$


From $[3,(3.17)]$

$$
\Sigma_{2} \leq\|\phi\|_{\infty}^{2} b^{-1}(a+1)(d-c) \sum_{j<j^{\prime}} a^{\left(j-j^{\prime}\right) / 2}=\|\phi\|_{\infty}^{2} b^{-1} \frac{(a+1)(d-c)}{(\sqrt{a}-1)} .
$$

Combining (7), (8) and Lemma 1, the conclusion follows.

The following lemma follows immediately as a corollary of Lemma 2 .

Lemma 3. Let $\phi(x)$ satisfy the hypotheses of Lemma 2, let $\alpha, \beta \in \mathcal{R}$ and $\rho(x):=$ $\phi(\alpha x+\beta)$. Then $\left\{\rho_{j, k}\right\}_{j, k \in \mathcal{Z}}$ is a Bessel sequence with Bessel bound

$$
\|\phi\|_{\infty}\left(\frac{\sqrt{a}}{\sqrt{a}-1}\right) \frac{(d-c)}{|\alpha|}\left\{\left[\frac{(d-c) b^{-1}}{|\alpha|}+1\right] V_{\phi}+\frac{(a+1)}{\sqrt{a}} b^{-1}\|\phi\|_{\infty}\right\} .
$$

In the sequel, we assume that $a=2$ and $b=1$. From Lemma 2, we also readily obtain

Lemma 4. Let $0<\varepsilon<\frac{1}{2}$, and let $\psi^{\{\varepsilon\}}(t):=\chi_{\left[\frac{1}{2}-\varepsilon, \frac{1}{2}\right)}(t)-\chi_{\left[\frac{1}{2}, \frac{1}{2}+\varepsilon\right)}(t)$. Then $\left\{\psi_{j, k}^{\{\varepsilon\}}\right\}_{j, k \in \mathcal{Z}}$ is a Bessel sequence with bound

$$
\frac{\sqrt{2} \varepsilon}{\sqrt{2}-1}(8 \varepsilon+4+3 / \sqrt{2})
$$

If $N_{m}(x)$ denotes the $m$-th order cardinal $B$-spline $([2,9])$, and

$$
g(t):=\chi_{[0, m-1]}(t) \sum_{k=0}^{m-2} N_{m}(t-k),
$$

then we have:

Lemma 5. Let $m \geq 2$. Then

(a) $g \in C^{(m-2)}[0, m-1]$,

(b) $g(0)=0$,

(c) $g(m-1)=1$,

(d) $\frac{d^{k}}{d x^{k}} g(0)=\frac{d^{k}}{d x^{k}} g(m-1)=0,1 \leq k \leq m-2$,

(e) $\int_{\mathcal{R}} g(t) d t=\frac{m-1}{2}$.

Proof. From, e.g., [2, Theorem 4.3] we know that

$$
N_{m}^{\prime}(x)=N_{m-1}(x)-N_{m-1}(x-1),
$$

and

$$
\operatorname{supp} N_{m}=[0, m],
$$

whence (a) and (b) follow.

Since (11) implies that $N_{m}(m-k-1)=0$ for $k<0$ or $k>m-2$, (c) follows from the identity ([2, Theorem 4.3])

$$
\sum_{k \in \mathcal{Z}} N_{m}(x-k)=1 .
$$

Applying (10) we have:

$$
\begin{aligned}
g^{\prime}(x) & =\chi_{[0, m-1]}(x) \sum_{k=0}^{m-2}\left[N_{m-1}(x-k)-N_{m-1}(x-k-1)\right] \\
& =\chi_{[0, m-1]}(x)\left[N_{m-1}(x)-N_{m-1}(x-m+1)\right],
\end{aligned}
$$

and (d) follows from (11). 
Finally, integrating by parts, and using $(11),(10)$ and $[2,(3.2 .16)]$ we have:

$$
\begin{aligned}
\sqrt{2 \pi} \hat{g}(x)= & \int_{0}^{m-1} e^{-i x t} g(t) d t \\
= & \left.(i / x) g(t) e^{-i t x}\right|_{0} ^{m-1}-(i / x) \int_{0}^{m-1} e^{-i t x} g^{\prime}(t) d t \\
= & (i / x) e^{(-m-1) x i} g(m-1) \\
& \quad-(i / x) \int_{0}^{m-1} e^{-i t x}\left[N_{m-1}(t)-N_{m-1}(t-m+1)\right] d t \\
= & (i / x) e^{-(m-1) x i}-(i / x) \int_{0}^{m-1} e^{-i t x} N_{m-1}(t) d t \\
= & (i / x) e^{-(m-1) x i}-(\sqrt{2 \pi} i / x) \hat{N}_{m-1}(x) \\
= & (i / x) e^{-(m-1) x i}-(i / x) e^{-(m-1) x i / 2}\left(\frac{\sin x / 2}{x / 2}\right)^{m-1} \\
= & (i / x) e^{-(m-1) x i / 2}\left[e^{-(m-1) x i / 2}-\left(\frac{\sin x / 2}{x / 2}\right)^{m-1}\right] .
\end{aligned}
$$

Separating the expression in brackets into real and imaginary parts, passing to the limit and noting that $\hat{g}(0)=\frac{1}{\sqrt{2 \pi}} \int_{0}^{m-1} g(t) d t$ is real, we conclude that $\sqrt{2 \pi} \hat{g}(0)=\frac{m-1}{2}$, i.e. $\int_{\mathcal{R}} g(t) d t=\frac{m-1}{2}$.

Lemma 6. Let $m \geq 2$ and

$$
q(t):=g(t-m+1)-\frac{1}{2} \sum_{k=0}^{m-2} N_{m}(t-k) .
$$

Then

(a) supp $q \subseteq[0,2 m-2]$,

(b) $q \in C^{m-2}(-\infty, 2 m-2]$,

(c) $q(0)=0$,

(d) $q(2 m-2)=1$,

(e) $\frac{d^{k}}{d x^{k}} q(0)=\frac{d^{k}}{d x^{k}} q(2 m-2)=0,1 \leq k \leq m-2$,

(f) $\int_{\mathcal{R}} q(t) d t=0$,

(g) $q \in B V(\mathcal{R})$ with $V_{q} \leq 2(m-1)$,

(h) $\|q\|_{\infty} \leq 1$.

Proof. (a), (b), (c), (d) and (e) follow from (10), (11) and Lemma 5. To prove (f), note that

$$
\begin{aligned}
\int_{\mathcal{R}} q(t) d t & =\int_{\mathcal{R}} g(t-m+1) d t-\frac{1}{2} \sum_{k=0}^{m-2} \int_{\mathcal{R}} N_{m}(t-k) d t \\
& \left.=\frac{m-1}{2}-\frac{1}{2} \sum_{k=0}^{m-2} \int_{\mathcal{R}} N_{m}(t-k) d t \quad \text { (by Lemma } 5(\mathrm{e})\right) \\
& =\frac{m-1}{2}-\frac{m-1}{2} \quad(\text { by }[2, \text { Theorem 4.3(i) }]) \\
& =0 .
\end{aligned}
$$


To prove $(\mathrm{g})$, note that on $[0, m-1], g(t-m+1)=0$, which implies that $g^{\prime}(t-m+1)=0$ for $0 \leq t \leq m-1$. Hence,

$$
q^{\prime}(t)=-(1 / 2) \sum_{k=0}^{m-2} N_{m}^{\prime}(t-k)=-(1 / 2)\left[N_{m-1}(t)-N_{m-1}(t-m+1)\right] .
$$

Thus for $0 \leq t \leq m-1,\left|q^{\prime}(t)\right| \leq \frac{1}{2}$, which implies that $V_{q}[0, m-1] \leq \frac{m-1}{2}$.

On $[m-1,2 m-2]$, we have

$$
\begin{aligned}
q^{\prime}(t) & =g^{\prime}(t-m+1)-\frac{1}{2} \sum_{k=0}^{m-2} N_{m}^{\prime}(t-k) \\
& =\left[N_{m-1}(t-m+1)-N_{m-1}(t-2 m+2)\right]+\frac{1}{2}\left[N_{m-1}(t-m+1)-N_{m-1}(t)\right] .
\end{aligned}
$$

Thus, $(m-1) \leq t \leq 2(m-1)$ implies that $\left|q^{\prime}(t)\right| \leq \frac{3}{2}$, and so $V_{q}[m-1,2 m-2] \leq$ $\frac{3}{2}(m-1)$. Hence,

$$
V_{q}(\mathcal{R})=V_{q}[0,2 m-2] \leq 2(m-1) .
$$

Finally, to prove (h) note that for $t \in \mathcal{R}$,

$$
0 \leq g(t-m+1) \leq 1 \text { and } 0 \leq \frac{1}{2} \sum_{k=0}^{m-2} N_{m}(t-k) \leq \frac{1}{2},
$$

implying that

$$
|q(t)|=\left|g(t-m+1)-\frac{1}{2} \sum_{k=0}^{m-2} N_{m}(t-k)\right| \leq 1,
$$

and the assertion follows.

Let

$$
\begin{gathered}
p_{1}^{\{\varepsilon\}}(x):=q\left(\frac{2 m-2}{\varepsilon}\{x+\varepsilon\}\right), \quad p_{2}^{\{\varepsilon\}}(x):=q\left(\frac{2 m-2}{\varepsilon}\{1+\varepsilon-x\}\right) \\
p_{3}^{\{\varepsilon\}}(x):=q\left(\frac{2 m-2}{\varepsilon}\left\{\frac{1}{2}-x\right\}\right), \quad p_{4}^{\{\varepsilon\}}(x):=q\left(\frac{2 m-2}{\varepsilon}\left\{x-\frac{1}{2}\right\}\right), \\
A^{\{\varepsilon\}}:=[-\varepsilon, 0) \cup\left(\frac{1}{2}-\varepsilon, \frac{1}{2}+\varepsilon\right) \cup(1,1+\varepsilon], \\
p^{\{\varepsilon\}}(x):=p_{1}^{\{\varepsilon\}}(x)-p_{2}^{\{\varepsilon\}}(x)+p_{3}^{\{\varepsilon\}}(x)-p_{4}^{\{\varepsilon\}}(x)-\psi^{\{\varepsilon\}}(x) .
\end{gathered}
$$

We have:

Lemma 7. Let $\varepsilon>0$. Then

(a) $\operatorname{supp} p^{\{\varepsilon\}} \subseteq A^{\{\varepsilon\}}$,

(b) $p^{\{\varepsilon\}} \in C^{m-2}\left(A^{\{\varepsilon\}}\right)$,

(c) $p^{\{\varepsilon\}}(-\varepsilon)=p^{\{\varepsilon\}}\left(\left(\frac{1}{2}-\varepsilon\right)^{-}\right)=p^{\{\varepsilon\}}\left(\left(\frac{1}{2}+\varepsilon\right)^{+}\right)=p^{\{\varepsilon\}}(1+\varepsilon)=0, p^{\{\varepsilon\}}\left(0^{-}\right)=1$, $p^{\{\varepsilon\}}\left(1^{+}\right)=-1$,

(d) For $1 \leq k \leq m-2$ we have

$$
\begin{aligned}
\frac{d^{k}}{d x^{k}} p^{\{\varepsilon\}}(-\varepsilon) & =\frac{d^{k}}{d x^{k}} p^{\{\varepsilon\}}\left(0^{-}\right)=\frac{d^{k}}{d x^{k}} p^{\{\varepsilon\}}\left(\left(\frac{1}{2}-\varepsilon\right)^{+}\right)=\frac{d^{k}}{d x^{k}} p^{\{\varepsilon\}}\left(\frac{1}{2}\right) \\
& =\frac{d^{k}}{d x^{k}} p^{\{\varepsilon\}}\left(\left(\frac{1}{2}+\varepsilon\right)^{-}\right)=\frac{d^{k}}{d x^{k}} p^{\{\varepsilon\}}\left(1^{+}\right)=\frac{d^{k}}{d x^{k}} p^{\{\varepsilon\}}(1+\varepsilon)=0,
\end{aligned}
$$


(e) $\left\{p_{j, k}^{\{\varepsilon\}}\right\}_{j, k \in \mathcal{Z}}$ is a Bessel sequence with bound

$$
\left\{4\left[\frac{\sqrt{2} \varepsilon}{(\sqrt{2}-1)}\left\{(\varepsilon+1)(2 m-2)+\frac{3}{\sqrt{2}}\right\}\right]^{\frac{1}{2}}+\left[\frac{\sqrt{2} \varepsilon}{(\sqrt{2}-1)}\{8 \varepsilon+4+3 / \sqrt{2}\}\right]^{\frac{1}{2}}\right\}^{2} .
$$

Proof. (a), (b), (c) and (d) follow from Lemma 6. By Lemmas 3 and 6, each of the functions $p_{i}^{\{\varepsilon\}}(x), 1 \leq i \leq 4$, generates a Bessel sequence with bound

$$
\frac{\sqrt{2} \varepsilon}{(\sqrt{2}-1)}\{(\varepsilon+1)(2 m-2)+3 / \sqrt{2}\}
$$

Applying Lemma 4 and Minkowski's inequality, (e) follows.

Theorem. Let $m \geq 2$ be an integer and let $\varepsilon>0$ be such that

$$
B:=\left\{4\left[\frac{\sqrt{2} \varepsilon}{(\sqrt{2}-1)}\left\{(\varepsilon+1)(2 m-2)+\frac{3}{\sqrt{2}}\right\}\right]^{\frac{1}{2}}+\left[\frac{\sqrt{2} \varepsilon}{(\sqrt{2}-1)}\left\{8 \varepsilon+4+\frac{3}{\sqrt{2}}\right\}\right]^{\frac{1}{2}}\right\}^{2}<1 .
$$

Let $h:=\chi_{\left[0, \frac{1}{2}\right]}-\chi_{\left[\frac{1}{2}, 1\right]}$ and $f^{\{\varepsilon\}}:=h+p^{\{\varepsilon\}}$. Then

(a) $f^{\{\varepsilon\}} \in C^{m-2}(-\infty, \infty)$,

(b) $\operatorname{supp} f^{\{\varepsilon\}} \subseteq[-\varepsilon, 1+\varepsilon]$,

(c) $\left\{f_{j, k}^{\{\varepsilon\}}\right\}_{j, k \in \mathcal{Z}}$ is a Riesz basis of $L^{2}(\mathcal{R})$ with frame bounds $(1-\sqrt{B})^{2}$ and $(1+\sqrt{B})^{2}$,

(d) If $0<\delta<\infty$, then $\left\|f^{\{\varepsilon\}}-h\right\|_{L^{\delta}(\mathcal{R})} \leq\left(1+2^{\delta}\right)^{1 / \delta}(2 \varepsilon)^{1 / \delta}$.

Proof. (a) and (b) follow from Lemma 7. Since the Haar system $\left\{h_{j, k}\right\}_{j, k \in \mathcal{Z}}$ is an orthogonal basis of $L^{2}(\mathcal{R})([2,4])$, (c) follows from Lemma 7 and [5, Theorem 5]. To prove $(\mathrm{d})$, note that $f^{\{\varepsilon\}}-h=p^{\{\varepsilon\}}$. Since the functions $p_{i}^{\{\varepsilon\}}, 1 \leq i \leq 4$, have disjoint support, Lemma 6 and the definition of $p^{\{\varepsilon\}}$ imply that $\left|p^{\{\varepsilon\}}(x)\right| \leq 1$ on $[-\varepsilon, 0] \cup[1,1+\varepsilon],\left|p^{\{\varepsilon\}}(x)\right| \leq 2$ on $[1 / 2-\varepsilon, 1 / 2+\varepsilon]$, and $\left|p^{\{\varepsilon\}}(x)\right|=0$ elsewhere.

\section{REFERENCES}

1. J. J. Benedetto and D. F. Walnut, Gabor frames for $L^{2}$ and related spaces, in "Wavelets: Mathematics and Applications" (J. J. Benedetto and M. W. Frazier, Eds.), pp. 97-162, CRC Press, Boca Raton, FL, 1994. MR 94i: 42040

2. C. K. Chui, "An Introduction to Wavelets", Academic Press, San Diego, 1992. MR 93f:42055

3. C. K. Chui and X. L. Shi, Bessel sequences and affine frames, Appl. Comput. Harm. Anal. 1 (1993), 29-49. MR 95b:42028

4. I. Daubechies, "Ten Lectures on Wavelets," SIAM, Philadelphia, 1992. MR 93e:42045

5. S. J. Favier and R. A. Zalik, On the stability of frames and Riesz bases, Appl. Comput. Harm. Anal. 2 (1995), 160-173. MR 96e:42030

6. K. Gröchenig, Acceleration of the frame algorithm, IEEE Trans. Signal Proc. 41 (1993), 3331-3340.

7. C. Houdré, Wavelets, probability and statistics: some bridges in "Wavelets: Mathematics and Applications" (J. J. Benedetto and M. W. Frazier, Eds), pp. 365-398, CRC Press, Boca Raton, FL 1994. MR 95c:60046

8. X. L. Shi, On $\wedge$ BMV functions with some applications to the theory of Fourier series, Sci. Sinica Ser. A 28 (1985), 147-158. MR 87a:42027

9. I. J. Schoenberg, "Cardinal Spline Interpolation", SIAM, Philadelphia, 1973. MR 54:8095 
10. G. Strang and T. Nguyen, "Wavelets and Filter Banks", Wellesley-Cambridge Press, Wellesley, Massachussetts, 1996. CMP 97:02

11. R. M. Young, "An Introduction to Nonharmonic Fourier Series", Academic Press, New York, 1980. MR 81m: 42027

Department of Mathematics, Auburn University, Auburn, Alabama 36849-5310

E-mail address: govilnk@mail.auburn.edu

E-mail address: zalik@mail.auburn.edu 